\title{
ALGEBRAIC STRUCTURE OF GRAPH OPERATIONS IN TERMS OF DEGREE SEQUENCES
}

\section{VISHNU NARAYAN MISHRA ${ }^{1,2 *}$, SADIK DELEN ${ }^{3}$ AND ISMAIL NACI CANGUL ${ }^{3}$}

${ }^{1}$ Department of Mathematics, Indira Gandhi National Tribal University, Lalpur, Amarkantak, Anuppur 484 887, Madhya Pradesh, India

${ }^{2}$ L. 1627 Awadh Puri Colony Beniganj, (Phase-III), Opposite - I.T.I., Ayodhya Main Road, Faizabad 224 001, Uttar Pradesh, India

${ }^{3}$ Dept. of Mathematics, Uludag University, Gorukle 16059 Bursa, Turkey

*Corresponding author vishnunarayanmishra@gmail.com

\begin{abstract}
In this paper, by means of the degree sequences (DS) of graphs and some graph theoretical and combinatorial methods, we determine the algebraic structure of the set of simple connected graphs according to two graph operations, namely join and Corona product. We shall conclude that in the case of join product, the set of graphs forms an abelian monoid whereas in the case of Corona product, this set is not even associative, it only satisfies two conditions, closeness and identity element. We also give a result on distributive law related to these two operations.
\end{abstract}

\section{INTRODUCTION}

${ }^{1,2}$ Let $G=(V(G), E(G))$ be a simple and connected graph with $|V(G)|=n$ vertices and $|E(G)|=m$ edges. Usually we use the notations $V$ and $E$ instead of $V(G)$ and $E(G)$, respectively. Here, by the word "simple", we mean that the graphs we consider do not have loops or multiple edges. Similar studies can be

Received 2017-11-27; accepted 2018-02-01; published 2018-11-02.

2010 Mathematics Subject Classification. 05C07, 05C10, 05C30, 05C76.

Key words and phrases. graph; degree sequence; join; Corona product; graph operation.

(C) 2018 Authors retain the copyrights of their papers, and all open access articles are distributed under the terms of the Creative Commons Attribution License. 
done for non-simple graphs as well.

For a vertex $v \in V$, we denote the degree of $v$ by $d_{G}(v)$, which is defined as the number of edges of $G$ meeting at $v$. A vertex with degree one is called a pendant vertex.

The notion of degree of a graph provides us an area to study various structural properties of graphs and hence attracts the attention of many graph theorists and also other scientists including chemists. If $d_{i}, 1 \leq i \leq n$, are the degrees of the vertices $v_{i}$ of a graph $G$ in any order, then the degree sequence (DS) of $G$ is the sequence $\left\{d_{1}, d_{2}, \cdots, d_{n}\right\}$. Also, in many papers, the DS is taken to be a non-decreasing sequence, whenever possible.

Conversely, a non-negative sequence $\left\{d_{1}, d_{2}, \cdots, d_{n}\right\}$ will be called realizable if it is the DS of any graph. It is clear from the definition that for a realizable DS, there is at least one graph having this DS. For example, the completely different two graphs in Figure 1 have the same DS.
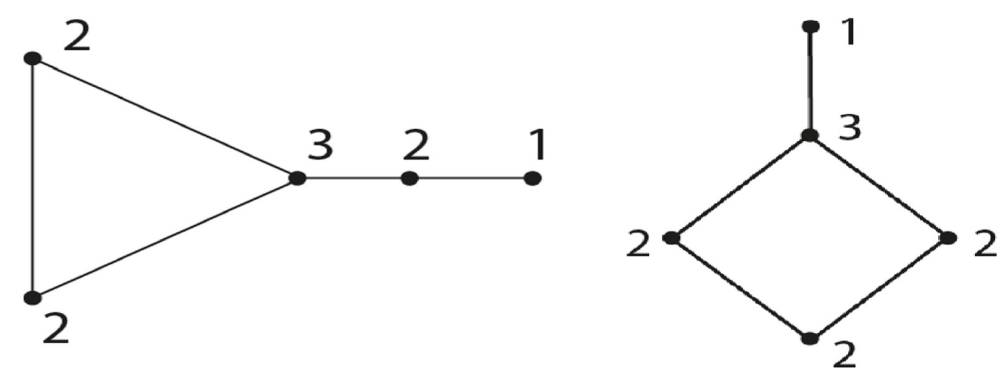

Fig. 1 Graphs with the same DS

For convenience and brevity, we shall denote the DS having repeated degrees with a shorter DS. For example, if the degree $d_{i}$ of the vertex $v_{i}$ appears $z_{i}$ times in the DS of a graph $G$, then we use $\left\{d_{1}^{\left(z_{1}\right)}, d_{2}^{\left(z_{2}\right)}, \cdots, d_{l}^{\left(z_{\ell}\right)}\right\}$ instead of $\left\{d_{1}, d_{2}, \cdots, d_{n}\right\}$ where $\ell \leq n$. Here the members $z_{i}$ are called the frequencies of the degrees. When $\ell=n$, that is, when all degrees are different, the DS is called perfect.

It is an open problem to determine that which DSs are realizable and there are several algorithms to determine that.

As usual, we denote by $P_{n}, C_{n}, S_{n}, K_{n}, T_{r, s}$ and $K_{r, s}$ the path, cycle, star, complete, complete bipartite and tadpole graphs, respectively, which are the most used graph examples in literature, see Figure 2. 

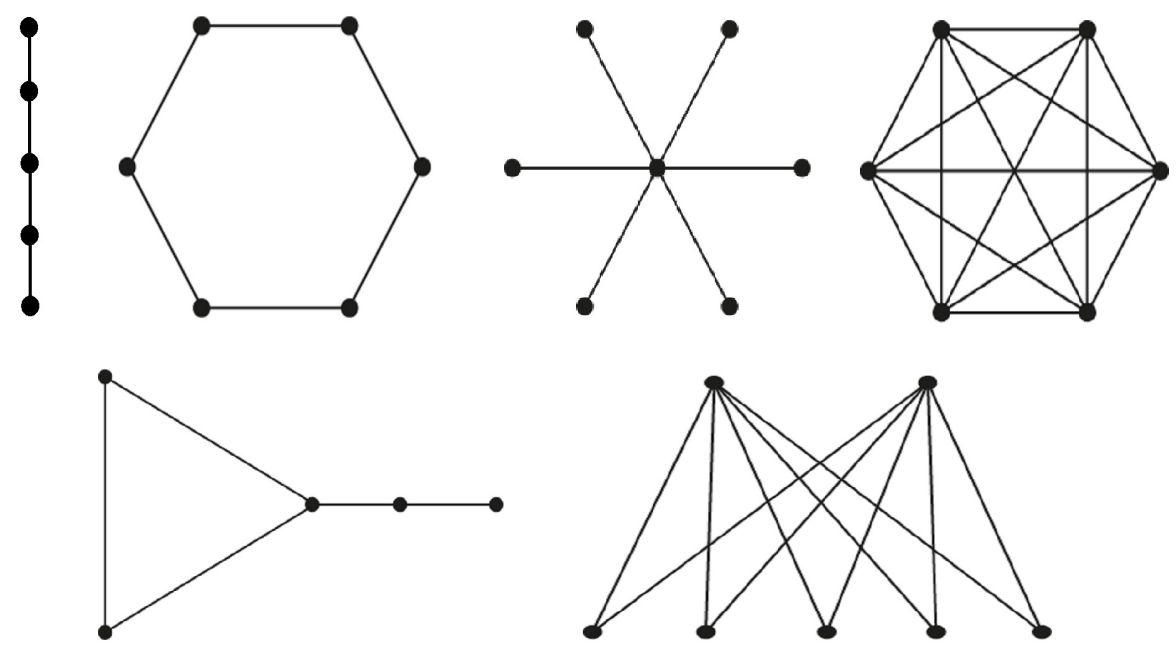

Fig. $2 P_{5}, C_{6}, S_{7}, K_{6}, T_{3,2}, K_{2,5}$

The number of vertices and edges of these well-known graph classes are given in Table 1.

TABLE 1. The number of vertices and edges of some graphs

\begin{tabular}{|c|c|c|}
\hline $\mathrm{G}$ & $\sharp$ vertices & $\sharp$ edges \\
\hline$P_{n}$ & $\mathrm{n}$ & $\mathrm{n}-1$ \\
\hline$C_{n}$ & $\mathrm{n}$ & $\mathrm{n}$ \\
\hline$S_{n}$ & $\mathrm{n}$ & $\mathrm{n}-1$ \\
\hline$K_{n}$ & $\mathrm{n}$ & $\left(\begin{array}{l}n \\
2\end{array}\right)$ \\
\hline$K_{r, s}$ & $\mathrm{r}+\mathrm{s}$ & $\mathrm{rs}$ \\
\hline$T_{r, s}$ & $\mathrm{r}+\mathrm{s}$ & $\mathrm{r}+\mathrm{s}$ \\
\hline
\end{tabular}

Another important reason to study the DSs of graphs is topological indices. A topological index (or a graph invariant) is a fixed invariant number for two isomorphic graphs and gives some information about the graph under consideration. These indices are especially useful in the study of molecular graphs. Some of the topological indices are defined by means of the vertex degrees: first and second Zagreb indices, first and second multiplicative Zagreb indices, atom-bond connectivity index, Narumi-Katayama index, geometricarithmetic index, harmonic index and sum-connectivity index etc. Therefore to know about the DS of the graph will help to obtain information about, e.g., the chemical properties of the graph. There are many papers on degree based topological indices, see e.g. [2]- [3]. 
The modern study of DSs started in 1981 by Bollobas, [1]. The same year, Tyshkevich et.al. established a correspondence between DS of a graph and some structural properties of this graph, [8]. In 1987, Tychkevich et.al. written a survey on the same correspondence, [9]. In [10], the authors gave a new version of the Erdös-Gallai theorem on the realizability of a given DS. In 2008, a new criterion on the same problem is given by Triphati and Tyagi, [7]. The same year, Kim et.al. gave a necessary and sufficient condition for the same problem, [5]. Ivanyi et.al, [4], gave an enumeration of DSs of simple graphs. Miller, [6] also gave a criteria for the realizability of given DSs.

There are several graph operations used in calculating some chemical invariants of graphs. Amongst these the join, cartesian, Corona product, union, disjunction, and symmetric difference are well-known. In this paper, after recalling two of these operations, join and Corona product, we shall determine the DS of these new product graphs and by means of these calculations, we shall study the algebraic properties of the join and Corona product of two graphs.

Let $G_{1}$ and $G_{2}$ be two graphs with $n_{1}$ and $n_{2}$ vertices and $m_{1}$ and $m_{2}$ edges, respectively. The join $G_{1} \vee G_{2}$ of graphs $G_{1}$ and $G_{2}$ with disjoint vertex sets $V\left(G_{1}\right)$ and $V\left(G_{2}\right)$ and edge sets $E\left(G_{1}\right)$ and $E\left(G_{2}\right)$ is the graph union $G_{1} \cup G_{2}$ together with all the edges joining $V\left(G_{1}\right)$ and $V\left(G_{2}\right)$. Thus, for example, $K_{p} \vee K_{q}=K_{p, q}$, the complete bipartite graph. We have $\left|V\left(G_{1} \vee G_{2}\right)\right|=n_{1}+n_{2}$ and $\left|E\left(G_{1} \vee G_{2}\right)\right|=m_{1}+m_{2}+n_{1} n_{2}$.

The Corona product $G_{1} \circ G_{2}$ of two graphs $G_{1}$ and $G_{2}$ is defined to be the graph $\Gamma$ obtained by taking one copy of $G_{1}$ (which has $n_{1}$ vertices) and $n_{1}$ copies of $G_{2}$, and then joining the $i$-th vertex of $G_{1}$ to every vertex in the $i$-th copy of $G_{2}$, for $i=1,2, \cdots, n_{1}$.

Let $G_{1}=\left(V_{1}, E_{1}\right)$ and $G_{2}=\left(V_{2}, E_{2}\right)$ be two graphs such that $V\left(G_{1}\right)=\left\{u_{1}, u_{2}, \cdots, u_{n_{1}}\right\},\left|E\left(G_{1}\right)\right|=m_{1}$ and $V\left(G_{2}\right)=\left\{v_{1}, v_{2}, \cdots v_{n_{2}}\right\},\left|E\left(G_{2}\right)\right|=m_{2}$. Then it follows from the definition of the Corona product that $G_{1} \circ G_{2}$ has $n_{1}\left(1+n_{2}\right)$ vertices and $m_{1}+n_{1} m_{2}+n_{1} n_{2}$ edges, where

$$
V\left(G_{1} \circ G_{2}\right)=\left\{\left(u_{i}, v_{j}\right), i=1,2, \ldots, n_{1} ; j=0,1,2, \ldots, n_{2}\right\}
$$

and

$$
\begin{aligned}
E\left(G_{1} \circ G_{2}\right) & =\left\{\left(\left(u_{i}, v_{0}\right),\left(u_{k}, v_{0}\right)\right),\left(u_{i}, u_{k}\right) \in E\left(G_{1}\right)\right\} \\
& \cup\left\{\left(\left(u_{i}, v_{j}\right),\left(u_{i}, v_{\ell}\right)\right),\left(v_{j}, v_{\ell}\right) \in E\left(G_{2}\right), i=1,2, \ldots, n_{1}\right\} \\
& \cup\left\{\left(\left(u_{i}, v_{0}\right),\left(u_{i}, v_{\ell}\right)\right), \ell=1,2, \ldots, n_{2}, i=1,2, \ldots, n_{1}\right\}
\end{aligned}
$$


It is clear that if $G_{1}$ is connected, then $G_{1} \circ G_{2}$ is connected, and in general $G_{1} \circ G_{2}$ is not isomorphic to $G_{2} \circ G_{1}$.

\section{Algebraic Properties of Join}

In this section, we deal with some algebraic properties of the join of two graphs. We shall try to determine the abstract algebraic structure of this new graph and also give the DS of the join graph $G_{1} \vee G_{2}$ of two graphs $G_{1}$ and $G_{2}$ where $G_{1}$ and $G_{2}$ are choosen from $P_{n}, C_{n}, S_{n}, K_{n}, T_{r, s}$ and $K_{r, s}$. In particular, in the case of join operation, the set of graphs forms an abelian monoid whereas in the case of Corona product, the set of graphs is not even associative, it only satisfies two conditions, closedness and identity element. It is clear to see that there are no zero divisors for both products.

Theorem 2.1. The DSs of all possible joins of the path, cycle, star, complete, tadpole and complete bipartite graphs are given in Table 2 .

Proof. We make the proof only for $P_{r} \vee P_{s}$ and $S_{r} \vee C_{s}$. Let $P_{r}=\left\{1^{(2)}, 2^{(r-2)}\right\}$ and $P_{s}=\left\{1^{(2)}, 2^{(s-2)}\right\}$. To visualize the situation, see Figure 3.

There are two types of vertices in each of $P_{r}$ and $P_{s}$. Therefore there are $2+2=4$ types of vertices in $P_{r} \vee P_{s}$. The first type is the two end vertices of $P_{r}$ (red ones) which are connected with the next green vertex in $P_{r}$ and $s$ vertices in $P_{s}$. Each of these two vertices add $s+1$ to the DS of $P_{r} \vee P_{s}$. Therefore they add $(s+1)^{(2)}$.

The second type of vertices are the mid ones in $P_{r}$ (green ones) each of which is connected to two neighboring vertices in $P_{r}$ and $s$ vertices in $P_{s}$. Each of these $r-2$ vertices adds $s+2$ to the DS of $P_{r} \vee P_{s}$. Therefore $(s+2)^{(r-2)}$ is added.

The third type of vertices are the two end vertices of $P_{s}$ (black ones) each of which is connected to one vertex in $P_{s}$ and $r$ vertices in $P_{r}$. They add $(r+1)^{2}$ to the DS of $P_{r} \vee P_{s}$. The fourth and last type of vertices are the mid-vertices (blue ones) in $P_{s}$. Their number is $s-2$ and each of which similarly adds $r+2$ to the DS of $P_{r} \vee P_{s}$. So their contribution is $(r+2)^{(s-2)}$. Therefore the required DS is

$$
P_{r} \vee P_{s}=\left\{(s+1)^{(2)},(s+2)^{(r-2)},(r+1)^{(2)},(r+2)^{(s-2)}\right\}
$$

Now we recall that $S_{r}=\left\{1^{(r-1)}, r-1\right\}$ and $C_{s}=\left\{2^{(s)}\right\}$. In Figure 4 , the join of these two graphs is drawn for $r=s=5$. 
TABLE 2. The DSs of the join of some well-known graph types

\begin{tabular}{|c|c|c|}
\hline$G_{1}$ & $G_{2}$ & $G_{1} \vee G_{2}$ \\
\hline$P_{r}$ & $P_{s}$ & $\left\{(s+1)^{(2)},(s+2)^{(r-2)},(r+1)^{(2)},(r+2)^{(s-2)}\right\}$ \\
\hline$P_{r}$ & $C_{s}$ & $\left\{(s+1)^{(2)},(s+2)^{(r-2)},(r+2)^{(s)}\right\}$ \\
\hline$P_{r}$ & $S_{s}$ & $\left\{(s+1)^{(2)},(s+2)^{(r-2)},(r+1)^{(s-1)}, r+s-1\right\}$ \\
\hline$P_{r}$ & $K_{s}$ & $\left\{(s+1)^{(2)},(s+2)^{(r-2)},(r+s-1)^{(s)}\right\}$ \\
\hline$P_{r}$ & $T_{s, t}$ & $\left\{(s+t+1)^{(2)},(s+t+2)^{(r-2)}, r+1,(r+2)^{(s+t-2)}, r+3\right\}$ \\
\hline$P_{r}$ & $K_{s, t}$ & $\left\{(s+t+1)^{(2)},(s+t+2)^{(r-2)},(r+s)^{(t)},(r+t)^{(s)}\right\}$ \\
\hline$C_{r}$ & $P_{s}$ & $\left\{(s+2)^{(r)},(r+1)^{(2)},(r+2)^{(s-2)}\right\}$ \\
\hline$C_{r}$ & $C_{s}$ & $\left\{(s+2)^{(r)},(r+2)^{(s)}\right\}$ \\
\hline$C_{r}$ & $S_{s}$ & $\left\{(s+2)^{(r)}, r+s-1,(r+1)^{(s-1)}\right\}$ \\
\hline$C_{r}$ & $K_{s}$ & $\left\{(s+2)^{(r)},(r+s-1)^{(s)}\right\}$ \\
\hline$C_{r}$ & $T_{s, t}$ & $\left\{(s+t+2)^{(r)}, r+1,(r+2)^{(s+t-2)}, r+3\right\}$ \\
\hline$C_{r}$ & $K_{s, t}$ & $\left\{(s+t+2)^{(r)},(r+s)^{(t)},(r+t)^{(s)}\right\}$ \\
\hline$S_{r}$ & $P_{s}$ & $\left\{(s+1)^{(r-1)}, r+s-1,(r+1)^{(2)},(r+2)^{(s-2)}\right\}$ \\
\hline$S_{r}$ & $C_{s}$ & $\left\{(s+1)^{(r-1)}, r+s-1,(r+2)^{(s)}\right\}$ \\
\hline$S_{r}$ & $S_{s}$ & $\left\{(s+1)^{(r-1)},(r+s-1)^{(2)},(r+1)^{(s-1)}\right\}$ \\
\hline$S_{r}$ & $K_{s}$ & $\left\{(s+1)^{(r-1)}, s+r-1,(r+s-1)^{(s)}\right\}$ \\
\hline$S_{r}$ & $T_{s, t}$ & $\left\{(s+t+1)^{(r-1)}, r+s+t-1, r+1,(r+2)^{(s+t-2)}, r+3\right\}$ \\
\hline$S_{r}$ & $K_{s, t}$ & $\left\{(s+t+1)^{(r-1)}, r+s+t-1,(r+s)^{(t)},(r+t)^{(s)}\right\}$ \\
\hline$K_{r}$ & $P_{s}$ & $\left\{(r+s-1)^{(r)},(r+1)^{(2)},(r+2)^{(s-2)}\right\}$ \\
\hline$K_{r}$ & $C_{s}$ & $\left\{(r+s-1)^{(r)},(r+2)^{(s)}\right\}$ \\
\hline$K_{r}$ & $S_{s}$ & $\left\{(r+s-1)^{(r)},(r+1)^{(s-1)}, r+s-1\right\}$ \\
\hline$K_{r}$ & $K_{s}$ & $\left\{(r+s-1)^{(r+s)}\right\}$ \\
\hline$K_{r}$ & $T_{s, t}$ & $\left\{(r+s+t-1)^{(r)}, r+1,(r+2)^{(s+t-2)}, r+3\right\}$ \\
\hline$K_{r}$ & $K_{s, t}$ & $\left\{(r+s+t-1)^{(r)},(r+s)^{(t)},(r+t)^{(s)}\right\}$ \\
\hline$T_{r, s}$ & $P_{t}$ & $\left\{(t+2)^{(r+s-2)}, t+1, t+3,(r+s+1)^{(2)},(r+s+2)^{(t-2)}\right\}$ \\
\hline$T_{r, s}$ & $C_{t}$ & $\left\{(t+2)^{(r+s-2)}, t+1, t+3,(r+s+2)^{(t)}\right\}$ \\
\hline$T_{r, s}$ & $S_{t}$ & $\left\{(t+2)^{(r+s-2)}, t+1, t+3,(r+s+1)^{(t-1)}, r+s+t-1\right\}$ \\
\hline$T_{r, s}$ & $K_{t}$ & $\left\{(t+2)^{(r+s-2)}, t+1, t+3,(r+s+t-1)^{(t)}\right\}$ \\
\hline$T_{r, s}$ & $T_{t, m}$ & $\begin{array}{c}(t+m+2)^{(r+s-2)}, t+m+1, t+m+3 \\
(r+s+2)^{(t+m-2)}, r+s+1, r+s+3\end{array}$ \\
\hline$T_{r, s}$ & $K_{t, m}$ & $\left\{(t+m+2)^{(r+s-2)}, t+m+1, t+m+3,(r+s+t)^{(m)},(r+s+m)^{(t)}\right\}$ \\
\hline$K_{r, s}$ & $P_{t}$ & $\left\{(r+t)^{(s)},(s+t)^{(r)},(r+s+1)^{(2)},(r+s+2)^{(t-2)}\right\}$ \\
\hline$K_{r, s}$ & $C_{t}$ & $\left\{(r+t)^{(s)},(s+t)^{(r)},(r+s+2)^{(t)}\right\}$ \\
\hline$K_{r, s}$ & $S_{t}$ & $\left\{(r+t)^{(s)},(s+t)^{(r)},(r+s+1)^{(t-1)}, r+s+t-1\right\}$ \\
\hline$K_{r, s}$ & $K_{t}$ & $\left\{(r+t)^{(s)},(s+t)^{(r)},(r+s+t-1)^{(t)}\right\}$ \\
\hline$K_{r, s}$ & $T_{t, m}$ & $\left\{(r+t+m)^{(s)},(s+t+m)^{(r)},(r+s+2)^{(t+m-2)}, r+s+1, r+s+3\right\}$ \\
\hline$K_{r, s}$ & $K_{t, m}$ & $\left\{(r+t+m)^{(s)},(s+t+m)^{(r)},(r+s+m)^{(t)},(r+s+t)^{(m)}\right\}$ \\
\hline
\end{tabular}


There are two types of vertices in star graph $S_{r}$ and only one type in cycle $C_{s}$. Therefore there are $2+1=3$ types of vertices in $S_{r} \vee C_{s}$. The first type of those is the end vertices (black ones) of the star graph $S_{r}$. Each of these is connected to the central vertex (blue coloured) by an edge in $S_{r}$ and to all $s$ vertices in $C_{s}$. Therefore each of these vertices adds $s+1$, and in total, $(s+1)^{(r-1)}$ is added to the DS. The second type is the unique central vertex in $S_{r}$ which is connected to $r-1$ end vertices in $S_{r}$ and also to all of $s$ vertices in $C_{s}$. It adds a total of $r+s-1$ to the DS. The third and final type of vertices is the $s$ vertices in $C_{s}$ (green ones). Each of them adds $r+2$ to the DS. Therefore a total of $(r+2)^{(s)}$ is added. Hence the DS of the required join graph is $S_{r} \vee C_{s}=\left\{(s+1)^{(r-1)}, r+s-1,(r+2)^{(s)}\right\}$.

Now we can study the algebraic structure of the set of graphs according to join operation:

Theorem 2.2. Let $G$ be the set of all simple connected graphs. Then $G$ is an abelian monoid with the join operation.

Proof. Let us have three graphs $G_{1}=\left\{\alpha_{11}^{\left(\beta_{11}\right)}, \cdots, \alpha_{1 \ell}^{\left(\beta_{1 \ell}\right)}\right\}, G_{2}=\left\{\alpha_{21}^{\left(\beta_{21}\right)}, \cdots, \alpha_{2 m}^{\left(\beta_{2 m}\right)}\right\}$ and $G_{3}=\left\{\alpha_{31}^{\left(\beta_{31}\right)}, \cdots, \alpha_{3 n}^{\left(\beta_{3 n}\right)}\right\}$ with the number of vertices $n_{1}, n_{2}, n_{3}$, respectively. We shall show that $G$ with the join operation is closed, associative, commutative, has identity element but no inverse elements:

First of all, the join of two simple connected graphs, by definition, is another simple connected graph, so $G$ is closed. For associativeness, note that

$$
\begin{aligned}
\left(G_{1} \vee G_{2}\right) \vee G_{3}=\{ & \left(n_{2}+\alpha_{11}\right)^{\left(\beta_{11}\right)}, \cdots,\left(n_{2}+\alpha_{1 \ell}\right)^{\left(\beta_{1 \ell}\right)}, \\
& \left.\left(n_{1}+\alpha_{21}\right)^{\left(\beta_{21}\right)}, \cdots,\left(n_{1}+\alpha_{2 m}\right)^{\left(\beta_{2 m}\right)}\right\} \vee\left\{\alpha_{31}^{\left(\beta_{31}\right)}, \alpha_{32}^{\left(\beta_{32}\right)}, \alpha_{33}^{\left(\beta_{33}\right)}\right\} \\
= & \left(n_{2}+n_{3}+\alpha_{11}\right)^{\left(\beta_{11}\right)}, \cdots,\left(n_{2}+n_{3}+\alpha_{1 \ell}\right)^{\left(\beta_{1 \ell}\right)} \\
& \left(n_{1}+n_{3}+\alpha_{21}\right)^{\left(\beta_{21}\right)}, \cdots,\left(n_{1}+n_{3}+\alpha_{2 m}\right)^{\left(\beta_{2 m}\right)} \\
& \left.\left(n_{1}+n_{2}+\alpha_{31}\right)^{\left(\beta_{31}\right)}, \cdots,\left(n_{1}+n_{2}+\alpha_{3 n}\right)^{\left(\beta_{3 n}\right)}\right\}
\end{aligned}
$$

and

$$
\begin{aligned}
G_{1} \vee\left(G_{2} \vee G_{3}\right)=\{ & \left.\alpha_{11}^{\left(\beta_{11}\right)}, \cdots, \alpha_{1 \ell}^{\left(\beta_{1 \ell}\right)}\right\} \vee\left\{\left(n_{3}+\alpha_{21}\right)^{\left(\beta_{21}\right)}, \cdots,\left(n_{3}+\alpha_{2 m}\right)^{\left(\beta_{2 m}\right)},\right. \\
& \left.\left(n_{2}+\alpha_{31}\right)^{\left(\beta_{31}\right)}, \cdots,\left(n_{2}+\alpha_{3 n}\right)^{\left(\beta_{3 n}\right)}\right\} \\
= & \left\{\left(n_{2}+n_{3}+\alpha_{11}\right)^{\left(\beta_{11}\right)}, \cdots,\left(n_{2}+n_{3}+\alpha_{1 \ell}\right)^{\left(\beta_{1 \ell}\right)}\right. \\
& \left(n_{1}+n_{3}+\alpha_{21}\right)^{\left(\beta_{21}\right)}, \cdots,\left(n_{1}+n_{3}+\alpha_{2 m}\right)^{\left(\beta_{2 m}\right)} \\
& \left.\left(n_{1}+n_{2}+\alpha_{31}\right)^{\left(\beta_{31}\right)}, \cdots,\left(n_{1}+n_{2}+\alpha_{3 n}\right)^{\left(\beta_{3 n}\right)}\right\}
\end{aligned}
$$




$$
\begin{aligned}
&=\left\{\left(n_{2}+n_{3}+\alpha_{11}\right)^{\left(\beta_{11}\right)}, \cdots,\left(n_{2}+n_{3}+\alpha_{1 \ell}\right)^{\left(\beta_{1 \ell}\right)},\right. \\
&\left(n_{1}+n_{3}+\alpha_{21}\right)^{\left(\beta_{21}\right)}, \cdots,\left(n_{1}+n_{3}+\alpha_{2 m}\right)^{\left(\beta_{2 m}\right)}, \\
&\left.\left(n_{1}+n_{2}+\alpha_{31}\right)^{\left(\beta_{31}\right)}, \cdots,\left(n_{1}+n_{2}+\alpha_{3 n}\right)^{\left(\beta_{3 n}\right)}\right\}
\end{aligned}
$$

therefore $G$ is associative. As

$$
\begin{aligned}
G_{1} \vee G_{2} & =\left\{\left(n_{2}+\alpha_{11}\right)^{\left(\beta_{11}\right)}, \cdots,\left(n_{2}+\alpha_{1 \ell}\right)^{\left(\beta_{1 \ell}\right)},\left(n_{1}+\alpha_{21}\right)^{\left(\beta_{21}\right)}, \cdots,\left(n_{1}+\alpha_{2 m}\right)^{\left(\beta_{2 m}\right)}\right\} \\
& =\left\{\left(n_{1}+\alpha_{21}\right)^{\left(\beta_{21}\right)}, \cdots,\left(n_{1}+\alpha_{2 m}\right)^{\left(\beta_{2 m}\right)},\left(n_{2}+\alpha_{11}\right)^{\left(\beta_{11}\right)}, \cdots,\left(n_{2}+\alpha_{1 \ell}\right)^{\left(\beta_{1 \ell}\right)}\right\} \\
& =G_{2} \vee G_{1},
\end{aligned}
$$

the operation is commutative. Therefore, to find the identity element, one needs to find a graph $Z$ with the property that $G_{1} \vee Z=G_{1}$. Let $Z=\left\{a_{1}^{b_{1}}, \cdots, a_{k}^{b_{k}}\right\}$ and let the graph $Z$ have $c$ vertices. Then $\left\{\alpha_{11}^{\left(\beta_{11}\right)}, \cdots, \alpha_{1 \ell}^{\left(\beta_{1 \ell}\right)}\right\} \vee\left\{a_{1}^{\left(b_{1}\right)}, \cdots, a_{k}^{\left(b_{k}\right)}\right\}=\left\{\alpha_{11}^{\left(\beta_{11}\right)}, \cdots, \alpha_{1 \ell}^{\left(\beta_{1 \ell}\right)}\right\}$ implies that

$$
\left\{\left(c+\alpha_{11}\right)^{\left(\beta_{11}\right)}, \cdots,\left(c+\alpha_{1 \ell}\right)^{\left(\beta_{1 \ell}\right)},\left(n_{1}+a_{1}\right)^{\left(b_{1}\right)}, \cdots,\left(n_{1}+a_{k}\right)^{\left(b_{k}\right)}\right\}=\left\{\alpha_{11}^{\left(\beta_{11}\right)}, \cdots, \alpha_{1 \ell}^{\left(\beta_{1 \ell}\right)}\right\}
$$

and this is only possible when $c=0$. For $c=0$, we have

$$
\left\{\alpha_{11}^{\left(\beta_{11}\right)}, \cdots, \alpha_{1 \ell}^{\left(\beta_{1 \ell}\right)},\left(n_{1}+a_{1}\right)^{\left(b_{1}\right)}, \cdots,\left(n_{1}+a_{k}\right)^{\left(b_{k}\right)}\right\}=\left\{\alpha_{11}^{\left(\beta_{11}\right)}, \cdots, \alpha_{1 \ell}^{\left(\beta_{1 \ell}\right)}\right\} .
$$

To have this equality, we must have

$$
\left(n_{1}+a_{1}\right)^{\left(b_{1}\right)}=0, \cdots,\left(n_{1}+a_{k}\right)^{\left(b_{k}\right)}=0 .
$$

Hence we must have no terms $\left(n_{1}+a_{1}\right)^{\left(b_{1}\right)}, \cdots,\left(n_{1}+a_{k}\right)^{\left(b_{k}\right)}$ in the DS of the identity element $Z$. This implies that $b_{1}=\cdots=b_{k}=0$. Therefore we can symbolically take $Z=\left\{1^{(0)}\right\}$ as the identity element.

Finally, let the inverse element of the graph $G_{1}=\left\{\alpha_{11}^{\left(\beta_{11}\right)}, \ldots, \alpha_{1 \ell}^{\left(\beta_{1 \ell}\right)}\right\}$ be denoted by $\left\{c_{1}^{\left(d_{1}\right)}, \ldots, c_{k}^{\left(d_{k}\right)}\right\}$. Let the number of vertices of the inverse element be $e$. Then

$$
G_{1} \vee\left\{c_{1}^{\left(d_{1}\right)}, \cdots, c_{k}^{\left(d_{k}\right)}\right\}=Z=\left\{1^{(0)}\right\}
$$

implies that

$$
\left\{\alpha_{11}^{\left(\beta_{11}\right)}, \cdots, \alpha_{1 \ell}^{\left(\beta_{1 \ell}\right)}\right\} \vee\left\{c_{1}^{\left(d_{1}\right)}, \cdots, c_{k}^{\left(d_{k}\right)}\right\}=\left\{1^{(0)}\right\}
$$

and therefore

$$
\left\{\left(e+\alpha_{11}\right)^{\left(\beta_{11}\right)}, \cdots,\left(e+\alpha_{1 \ell}\right)^{\left(\beta_{1 \ell}\right)},\left(n_{1}+c_{1}\right)^{\left(d_{1}\right)}, \cdots,\left(n_{1}+c_{k}\right)^{\left(d_{k}\right)}\right\}=\left\{1^{(0)}\right\} .
$$

As there is no solution to that equation, we conclude that there is no inverse element for the join operation. Therefore the result follows. 


\section{Algebraic Properties of Corona Product}

Theorem 3.1. The DSs of all possible Corona products of the path, cycle, star, complete, tadpole and complete bipartite graphs are given in Table 3.

Theorem 3.2. Let $G$ be the set of all simple connected graphs. Then $G$ with Corona product operation is closed with identity.

Proof. First, by the definition of the operation, $G$ is closed. Secondly, for associativeness, we should note that

$$
\begin{aligned}
&\left(G_{1} \circ G_{2}\right) \circ G_{3}=\left\{\left(n_{2}+\alpha_{11}\right)^{\left(\beta_{11}\right)}, \cdots,\left(n_{2}+\alpha_{1 \ell}\right)^{\left(\beta_{1 \ell}\right)},\right. \\
&\left.\left(1+\alpha_{21}\right)^{\left(n_{1} \beta_{21}\right)}, \cdots,\left(1+\alpha_{2 m}\right)^{\left(n_{1} \beta_{2 m}\right)}\right\} \circ\left\{\alpha_{31}^{\left(\beta_{31}\right)}, \cdots, \alpha_{3 n}^{\left(\beta_{3 n}\right)}\right\} \\
&=\{\left(n_{2}+n_{3}+\alpha_{11}\right)^{\left(\beta_{11}\right)}, \cdots,\left(n_{2}+n_{3}+\alpha_{1 \ell}\right)^{\left(\beta_{1 \ell}\right)}, \\
&\left(1+n_{3}+\alpha_{21}\right)^{\left(n_{1} \beta_{21}\right)}, \cdots,\left(1+n_{3}+\alpha_{2 m}\right)^{\left(n_{1} \beta_{2 m}\right)}, \\
&\left.\left(1+\alpha_{31}\right)^{\left(n_{1}\left(n_{1}+n_{2}\right) \beta_{31}\right)}, \cdots,\left(1+\alpha_{3 n}\right)^{\left(n_{1}\left(n_{1}+n_{2}\right) \beta_{3 n}\right)}\right\}
\end{aligned}
$$

and

$$
\begin{aligned}
& G_{1} \circ\left(G_{2} \circ G_{3}\right)=\left\{\alpha_{11}^{\left(\beta_{11}\right)}, \cdots, \alpha_{1 \ell}^{\left(\beta_{1 \ell}\right)}\right\} \circ\left\{\left(n_{3}+\alpha_{21}\right)^{\left(\beta_{21}\right)}, \cdots,\left(n_{3}+\alpha_{2 m}\right)^{\beta_{2 m}},\right. \\
&\left.\left(1+\alpha_{31}\right)^{\left(n_{2} \beta_{31}\right)}, \cdots,\left(1+\alpha_{3 n}\right)^{\left(n_{2} \beta_{3 n}\right)}\right\} \\
&=\{\left(n_{2}+n_{2} n_{3}+\alpha_{11}\right)^{\left(\beta_{11}\right)}, \cdots,\left(n_{2}+n_{2} n_{3}+\alpha_{1 \ell}\right)^{\left(\beta_{1 \ell}\right)} \\
&\left(1+n_{3}+\alpha_{21}\right)^{\left(n_{1} \beta_{21}\right)}, \cdots,\left(1+n_{3}+\alpha_{2 m}\right)^{\left(n_{1} \beta_{2 m}\right)} \\
&\left.\left(2+\alpha_{31}\right)^{\left(n_{1} n_{2} \beta_{31}\right)}, \cdots,\left(2+\alpha_{3 n}\right)^{\left(n_{1} n_{2} \beta_{3 n}\right)}\right\} .
\end{aligned}
$$

That is, $G$ is not associative.

For the identity element, we should find a graph $Z$ such that $G_{1} \circ Z=G_{1}$. Let $Z=\left\{a_{1}^{\left(b_{1}\right)}, \cdots, a_{k}^{\left(b_{k}\right)}\right\}$ and let the number of vertices of $Z$ be $c$. We have

$$
\left\{\alpha_{11}^{\left(\beta_{11}\right)}, \cdots, \alpha_{1 \ell}^{\left(\beta_{1 \ell}\right)}\right\} \circ\left\{a_{1}^{\left(b_{1}\right)}, \cdots, a_{k}^{\left(b_{k}\right)}\right\}=\left\{\alpha_{11}^{\left(\beta_{11}\right)}, \cdots, \alpha_{1 \ell}^{\left(\beta_{1 \ell}\right)}\right\}
$$

and hence, we get

$$
\left\{\left(c+\alpha_{11}\right)^{\left(\beta_{11}\right)}, \cdots,\left(c+\alpha_{1 \ell}\right)^{\left(\beta_{1 \ell}\right)},\left(1+a_{1}\right)^{\left(n_{1} b_{1}\right)}, \cdots,\left(1+a_{k}\right)^{\left(n_{1} b_{k}\right)}\right\}=\left\{\alpha_{11}^{\left(\beta_{11}\right)}, \cdots, \alpha_{1 \ell}^{\left(\beta_{1 \ell}\right)}\right\} .
$$

For this equation to have a solution, we must have $c=0$ and also $b_{1}, \cdots, b_{k}=0$. For the sake of brevity, if we take 1 instead of $1+a_{1}, \cdots, 1+a_{k}$, we conclude that $Z=\left\{1^{(0)}\right\}$ is the required identity element. Let 
TABLE 3. The DSs of the Corona product of well-known graph types

\begin{tabular}{|c|c|c|}
\hline$G_{1}$ & $G_{2}$ & $G_{1} \circ G_{2}$ \\
\hline$G_{1}$ & $G_{2}$ & $G_{1} \circ G_{2}$ \\
\hline$P_{r}$ & $P_{s}$ & $\left\{2^{(2 r)}, 3^{(r(s-2))},(s+1)^{(2)},(s+2)^{(r-2)}\right\}$ \\
\hline$P_{r}$ & $C_{s}$ & $\left\{3^{(r s)},(s+1)^{(2)},(s+2)^{(r-2)}\right\}$ \\
\hline$P_{r}$ & $S_{s}$ & $\left\{2^{(r(s-1))}, s^{(r)},(s+1)^{(2)},(s+2)^{(r-2)}\right\}$ \\
\hline$P_{r}$ & $K_{s}$ & $\left\{s^{(r s)},(s+1)^{(2)},(s+2)^{(r-2)}\right\}$ \\
\hline$P_{r}$ & $T_{s, t}$ & $\left\{2^{(r)}, 3^{(r(s+t-2))}, 4^{(r)},(s+t+1)^{(2)},(s+t+2)^{(r-2)}\right\}$ \\
\hline$P_{r}$ & $K_{s, t}$ & $\left\{(s+1)^{(r t)},(t+1)^{(r s)},(s+t+1)^{(2)},(s+t+2)^{(r-2)}\right\}$ \\
\hline$C_{r}$ & $P_{s}$ & $\left\{2^{(2 r)}, 3^{(r(s-2))},(s+2)^{(r)}\right\}$ \\
\hline$C_{r}$ & $C_{s}$ & $\left\{3^{(r s)},(s+2)^{(r)}\right\}$ \\
\hline$C_{r}$ & $S_{s}$ & $\left\{2^{(r(s-1))}, s^{(r)},(s+2)^{(r)}\right\}$ \\
\hline$C_{r}$ & $K_{s}$ & $\left\{s^{(r s)},(s+2)^{(r)}\right\}$ \\
\hline$C_{r}$ & $T_{s, t}$ & $\left\{2^{(r)}, 3^{(r(s+t-2))}, 4^{(r)},(s+t+2)^{(r)}\right\}$ \\
\hline$C_{r}$ & $K_{s, t}$ & $\left\{(s+1)^{(r t)},(t+1)^{(r s)},(s+t+2)^{(r)}\right\}$ \\
\hline$S_{r}$ & $P_{s}$ & $\left\{2^{(2 r)}, 3^{(r(s-2))},(s+1)^{(r-1)}, r+s-1\right\}$ \\
\hline$S_{r}$ & $C_{s}$ & $\left\{3^{(r s)},(s+1)^{(r-1)}, r+s-1\right\}$ \\
\hline$S_{r}$ & $S_{s}$ & $\left\{2^{(r(s-1))}, s^{(r)},(s+1)^{(r-1)}, r+s-1\right\}$ \\
\hline$S_{r}$ & $K_{s}$ & $\left\{s^{(r s)},(s+1)^{(r-1)}, r+s-1\right\}$ \\
\hline$S_{r}$ & $T_{s, t}$ & $\left\{2^{(r)}, 3^{(r(s+t-2))}, 4^{(r)},(s+t+1)^{(r-1)}, r+s+t-1\right\}$ \\
\hline$S_{r}$ & $K_{s, t}$ & $\left\{(s+1)^{(r t)},(t+1)^{(r s)},(s+t+1)^{(r-1)}, r+s+t-1\right\}$ \\
\hline$K_{r}$ & $P_{s}$ & $\left\{2^{(2 r)}, 3^{(r(s-2))},(r+s-1)^{(r)}\right\}$ \\
\hline$K_{r}$ & $C_{s}$ & $\left\{3^{(r s)},(r+s-1)^{(r)}\right\}$ \\
\hline$K_{r}$ & $S_{s}$ & $\left\{2^{(r(s-1))}, s^{(r)},(r+s-1)^{(r)}\right\}$ \\
\hline$K_{r}$ & $K_{s}$ & $\left\{s^{(r s)},(r+s-1)^{(r)}\right\}$ \\
\hline$K_{r}$ & $T_{s, t}$ & $\left\{2^{(r)}, 3^{(r(s+t-2))}, 4^{(r)},(r+s+t-1)^{(r)}\right\}$ \\
\hline$K_{r}$ & $K_{s, t}$ & $\left\{(s+1)^{(r t)},(t+1)^{(r s)},(r+s+t-1)^{(r)}\right\}$ \\
\hline$T_{r, s}$ & $P_{t}$ & $\left\{2^{(2(r+s))}, 3^{((r+s)(t-2))}, t+1,(t+2)^{(r+s-2)}, t+3\right\}$ \\
\hline$T_{r, s}$ & $C_{t}$ & $\left\{3^{((r+s) t)}, t+1,(t+2)^{(r+s-2)}, t+3\right\}$ \\
\hline$T_{r, s}$ & $S_{t}$ & $\left\{2^{((r+s)(t-1))}, t^{(r+s)}, t+1,(t+2)^{(r+s-2)}, t+3\right\}$ \\
\hline$T_{r, s}$ & $K_{t}$ & $\left\{t^{((r+s) t)}, t+1,(t+2)^{(r+s-2)}, t+3\right\}$ \\
\hline$T_{r, s}$ & $T_{t, m}$ & $\left\{\begin{array}{c}2^{(r+s)}, 3^{((r+s)(t+m-2))}, 4^{(r+s)}, t+m+1 \\
(t+m+2)^{(r+s-2)}, t+m+3\end{array}\right.$ \\
\hline$T_{r, s}$ & $K_{t, m}$ & $\left\{\begin{array}{c}(t+1)^{((r+s) m)},(m+1)^{((r+s) t)}, t+m+1 \\
(t+m+2)^{(r+s-2)}, t+m+3\end{array}\right.$ \\
\hline$K_{r, s}$ & $P_{t}$ & $\left\{2^{(2(r+s))}, 3^{((r+s)(t-2))},(r+t)^{(s)},(s+t)^{(r)}\right\}$ \\
\hline$K_{r, s}$ & $C_{t}$ & $\left\{3^{((r+s) t)},(r+t)^{(s)},(s+t)^{(r)}\right\}$ \\
\hline$K_{r, s}$ & $S_{t}$ & $\left\{2^{((r+s)(t-1))}, t^{(r+s)},(r+t)^{(s)},(s+t)^{(r)}\right\}$ \\
\hline$K_{r, s}$ & $K_{t}$ & $\left\{t^{((r+s) t)},(r+t)^{(s)},(s+t)^{(r)}\right\}$ \\
\hline$K_{r, s}$ & $T_{t, m}$ & $\left\{2^{(r+s)}, 3^{((r+s)(t+m-2))}, 4^{(r+s)},(r+t+m)^{(s)},(s+t+m)^{(r)}\right\}$ \\
\hline$K_{r, s}$ & $K_{t, m}$ & $\left\{(t+1)^{((r+s) m)},(m+1)^{((r+s) t)},(r+t+m)^{(s)},(s+t+m)^{(r)}\right\}$ \\
\hline
\end{tabular}


$T=\left\{c_{1}^{\left(d_{1}\right)}, \cdots, c_{k}^{\left(d_{k}\right)}\right\}$ be the inverse element of a graph $G_{1}=\left\{\alpha_{11}^{\left(\beta_{11}\right)}, \cdots, \alpha_{1 \ell}^{\left(\beta_{1 \ell}\right)}\right\}$. Then they must satisfy the equation $G_{1} \circ\left\{c_{1}^{\left(d_{1}\right)}, \cdots, c_{k}^{\left(d_{k}\right)}\right\}=Z=\left\{1^{(0)}\right\}$. If the number of vertices of $T$ is $e$, then we have

$$
\left\{\alpha_{11}^{\left(\beta_{11}\right)}, \cdots, \alpha_{1 \ell}^{\left(\beta_{1 \ell}\right)}\right\} \circ\left\{c_{1}^{\left(d_{1}\right)}, \cdots, c_{k}^{\left(d_{k}\right)}\right\}=\left\{1^{(0)}\right\}
$$

In this case, this equation cannot be hold, implying that there is no inverse element in $G$.

Finally, as

$$
\begin{aligned}
\left\{\alpha_{11}^{\left(\beta_{11}\right)}, \cdots, \alpha_{1 \ell}^{\left(\beta_{1 \ell}\right)}\right\} \circ\left\{\alpha_{21}^{\left(\beta_{21}\right)}, \cdots, \alpha_{2 m}^{\beta_{2 m}}\right\}= & \left\{\left(n_{2}+\alpha_{11}\right)^{\left(\beta_{11}\right)}, \cdots,\left(n_{2}+\alpha_{1 \ell}\right)^{\left(\beta_{1 \ell}\right)},\right. \\
& \left.\left(1+\alpha_{21}\right)^{\left(n_{1} \beta_{21}\right)}, \cdots,\left(1+\alpha_{2 m}\right)^{\left(n_{1} \beta_{2 m}\right)}\right\}
\end{aligned}
$$

and

$$
\begin{aligned}
\left\{\alpha_{21}^{\left(\beta_{21}\right)}, \cdots, \alpha_{2 m}^{\left(\beta_{2 m}\right)}\right\} \circ\left\{\alpha_{11}^{\left(\beta_{11}\right)}, \cdots, \alpha_{1 \ell}^{\left(\beta_{1 \ell}\right)}\right\}= & \left\{\left(n_{1}+\alpha_{21}\right)^{\left(\beta_{21}\right)}, \cdots,\left(n_{1}+\alpha_{2 m}\right)^{\left(\beta_{2 m}\right)},\right. \\
& \left.\left(1+\alpha_{11}\right)^{\left(n_{2} \beta_{11}\right)}, \cdots,\left(1+\alpha_{1 \ell}\right)^{\left(n_{2} \beta_{1 \ell}\right)}\right\},
\end{aligned}
$$

$G$ can only be commutative when $G_{1}=G_{2}$. In general, $G$ is not commutative.

Finally, we check whether the distributive law holds when we have join and Corona in place of $\cdot$ and + , or vice versa:

Theorem 3.3. Neither join nor Corona operation is not distributive on each other. That is

(i) $G_{1} \vee\left(G_{2} \circ G_{3}\right) \neq\left(G_{1} \vee G_{2}\right) \circ\left(G_{1} \vee G_{3}\right)$,

(ii) $G_{1} \circ\left(G_{2} \vee G_{3}\right) \neq\left(G_{1} \circ G_{2}\right) \vee\left(G_{1} \circ G_{3}\right)$.

Proof. Both claims follow after the following calculations:

(i) $G_{1} \vee\left(G_{2} \circ G_{3}\right)=\left\{\alpha_{11}^{\left(\beta_{11}\right)}, \cdots, \alpha_{1 \ell}^{\left(\beta_{1 \ell}\right)}\right\} \vee\left\{\left(n_{3}+\alpha_{21}\right)^{\left(\beta_{21}\right)}, \cdots,\left(n_{3}+\alpha_{2 m}\right)^{\left(\beta_{2 m}\right)}\right.$,

$$
\begin{aligned}
&\left.\left(1+\alpha_{31}\right)^{\left(n_{2} \beta_{31}\right)}, \cdots,\left(1+\alpha_{3 n}\right)^{\left(n_{2} \beta_{3 n}\right)}\right\} \\
&=\left\{\left(n_{2}+n_{2} n_{3}+\alpha_{11}\right)^{\left(\beta_{11}\right)}, \cdots,\left(n_{2}+n_{2} n_{3}+\alpha_{1 \ell}\right)^{\left(\beta_{1 \ell}\right)},\right. \\
&\left(n_{1}+n_{3}+\alpha_{21}\right)^{\left(\beta_{21}\right)}, \cdots,\left(n_{1}+n_{3}+\alpha_{2 m}\right)^{\left(\beta_{2 m}\right)}, \\
&\left.\left(1+n_{1}+\alpha_{31}\right)^{\left(n_{2} \beta_{31}\right)}, \cdots,\left(1+n_{1}+\alpha_{3 n}\right)^{\left(n_{2} \beta_{3 n}\right)}\right\}
\end{aligned}
$$


and

$$
\begin{aligned}
\left(G_{1} \vee G_{2}\right) \circ\left(G_{1} \vee G_{3}\right)= & \left\{\left(n_{2}+\alpha_{11}\right)^{\left(\beta_{11}\right)}, \cdots,\left(n_{2}+\alpha_{1 \ell}\right)^{\left(\beta_{1 \ell}\right)},\left(n_{1}+\alpha_{21}\right)^{\left(\beta_{21}\right)}, \cdots,\right. \\
& \left.\left(n_{1}+\alpha_{2 m}\right)^{\left(\beta_{2 m}\right)}\right\} \circ\left\{\left(n_{3}+\alpha_{11}\right)^{\left(\beta_{11}\right)}, \cdots,\left(n_{3}+\alpha_{1 \ell}\right)^{\left(\beta_{1 \ell}\right)},\right. \\
\left.\left(n_{1}+\alpha_{31}\right)^{\left(\beta_{31}\right)}, \cdots,\left(n_{1}+\alpha_{3 n}\right)^{\left(\beta_{3 n}\right)}\right\} & \\
= & \left(n_{1}+n_{2}+n_{3}+\alpha_{11}\right)^{\left(\beta_{11}\right)}, \cdots,\left(n_{1}+n_{2}+n_{3}+\alpha_{1 \ell}\right)^{\left(\beta_{1 \ell}\right)}, \\
& \left(2 n_{1}+n_{3}+\alpha_{21}\right)^{\left(\beta_{21}\right)}, \cdots,\left(2 n_{1}+n_{3}+\alpha_{2 m}\right)^{\left(\beta_{2 m}\right)}, \\
& \left(1+n_{3}+\alpha_{11}\right)^{\left(n_{1}+n_{2}\right) \beta_{11}}, \cdots,\left(1+n_{3}+\alpha_{1 \ell}\right)^{\left(\left(n_{1}+n_{2}\right) \beta_{1 \ell}\right)}, \\
& \left.\left(1+n_{1}+\alpha_{31}\right)^{\left(\left(n_{1}+n_{2}\right) \beta_{31}\right)}, \cdots,\left(1+n_{1}+\alpha_{3 n}\right)^{\left(\left(n_{1}+n_{2}\right) \beta_{3 n}\right)}\right\} .
\end{aligned}
$$

$($ ii $) G_{1} \circ\left(G_{2} \vee G_{3}\right)=\left\{\alpha_{11}^{\left(\beta_{11}\right)}, \cdots, \alpha_{1 \ell}^{\left(\beta_{1 \ell}\right)}\right\} \circ\left\{\left(n_{3}+\alpha_{21}\right)^{\left(\beta_{21}\right)}, \cdots,\left(n_{3}+\alpha_{2 m}\right)^{\left(\beta_{2 m}\right)}\right.$,

$$
\begin{aligned}
&\left.\left(n_{2}+\alpha_{31}\right)^{\left(\beta_{31}\right)}, \cdots,\left(n_{2}+\alpha_{3 n}\right)^{\left(\beta_{3 n}\right)}\right\} \\
&=\left\{\left(n_{2}+n_{3}+\alpha_{11}\right)^{\left(\beta_{11}\right)}, \cdots,\left(n_{2}+n_{3}+\alpha_{1 \ell}\right)^{\left(\beta_{1 \ell}\right)},\right. \\
& \\
&\left(1+n_{3}+\alpha_{21}\right)^{\left(n_{1} \beta_{21}\right)}, \cdots,\left(1+n_{3}+\alpha_{2 m}\right)^{\left(n_{1} \beta_{2 m}\right)}, \\
& \\
&\left.\left(1+n_{2}+\alpha_{31}\right)^{\left(n_{1} \beta_{31}\right)}, \cdots,\left(1+n_{2}+\alpha_{3 n}\right)^{\left(n_{1} \beta_{3 n}\right)}\right\}
\end{aligned}
$$

and also

$$
\begin{aligned}
\left(G_{1} \circ G_{2}\right) \vee\left(G_{1} \circ G_{3}\right)= & \left\{\left(n_{2}+\alpha_{11}\right)^{\left(\beta_{11}\right)}, \cdots,\left(n_{2}+\alpha_{1 \ell}\right)^{\left(\beta_{1 \ell}\right)},\left(1+\alpha_{21}\right)^{\left(n_{1} \beta_{21}\right)}, \cdots,\right. \\
& \left.\left(1+\alpha_{2 m}\right)^{\left(n_{1} \beta_{2 m}\right)}\right\} \vee\left\{\left(n_{3}+\alpha_{11}\right)^{\left(\beta_{11}\right)}, \cdots,\left(n_{3}+\alpha_{1 \ell}\right)^{\left(\beta_{1 \ell}\right)},\right. \\
\left.\left(1+\alpha_{31}\right)^{\left(n_{1} \beta_{31}\right)}, \cdots,\left(1+\alpha_{3 n}\right)^{\left(n_{1} \beta_{3 n}\right)}\right\} & \\
= & \left(n_{1}+n_{1} n_{3}+n_{2}+\alpha_{11}\right)^{\beta_{11}}, \cdots,\left(n_{1}+n_{1} n_{3}+n_{2}+\alpha_{1 \ell}\right)^{\left(\beta_{1 \ell}\right)}, \\
& \left(1+n_{1}+n_{1} n_{3}+\alpha_{21}\right)^{\left(n_{1} \beta_{21}\right)}, \cdots,\left(1+n_{1}+n_{1} n_{3}+\alpha_{2 m}\right)^{\left(n_{1} \beta_{2 m}\right)}, \\
& \left(n_{1}+n_{1} n_{2}+n_{3}+\alpha_{11}\right)^{\beta_{11}}, \cdots,\left(n_{1}+n_{1} n_{2}+n_{3}+\alpha_{1 \ell}\right)^{\left(\beta_{1 \ell}\right)}, \\
& \left.\left(1+n_{1}+n_{1} n_{2}+\alpha_{31}\right)^{\left(n_{1} \beta_{31}\right)}, \cdots,\left(1+n_{1}+n_{1} n_{2}+\alpha_{3 n}\right)^{\left(n_{1} \beta_{3 n}\right)}\right\} .
\end{aligned}
$$




\section{REFERENCES}

[1] B. Bollobas, Degree Sequences of Random Graphs, Discrete Math. 33 (1981), 1-19.

[2] K. C. Das, N. Akgunes, M. Togan, A. Yurttas, I. N. Cangul, A. S. Cevik, On the first Zagreb index and multiplicative Zagreb coindices of graphs, An. tiin. Univ. Ovidius Constana, Ser. Mat. 24 (1) (2016), 153-176.

[3] K. C. Das, A. Yurttas, M. Togan, I. N. Cangul, A. S. Cevik, The Multiplicative Zagreb Indices of Graph Operations, J. Inequal. Appl. 90 (2013), 1-14.

[4] A. Ivanyi, L. Lucz, G. Gombos, T. Matuszka, Parallel Enumeration of Degree Sequences of Simple Graphs II, Acta Univ. Sapientiae, Informatica 5 (2) (2013), 245-270.

[5] H. Kim, Z. Toroczkai, I. Miklos, P. L. Erdös, L. A. Szekely, On Realizing all Simple Graphs with a Given Degree Sequence, J. Phys. A: Math. Theor. 42 (2009), 1-6.

[6] J. W. Miller, Reduced Criteria for Degree Sequences, Discrete Math. 313 (2013), 550-562.

[7] A. Triphati, H. Tyagi, A Simple Criterion on Degree Sequences of Graphs, Discrete Appl. Math. 156 (2008), 3513-3517.

[8] R. I. Tyshkevich, O. I. Mel'nikov, V. M. Kotov, On Graphs and Degree Sequences: Canonical Decomposition, Kibernetika 6 (1981), 5-8.

[9] R. I. Tyshkevich, A. A. Chernyak, Zh. A. Chernyak, Graphs and Degree Sequences I, Cybernetics 23 (6) (1987), $734-745$.

[10] I. E. Zverovich, V. E. Zverovich, Contributions to the Theory of Graphic Sequences, Discrete Math. 105 (1992), $293-303$. 